\title{
Imaging spectroscopy of solar pores
}

\author{
J. Hirzberger ${ }^{\star}$ \\ Institut für Geophysik, Astrophysik und Meteorologie, Universitätsplatz 5, 8010 Graz, Austria \\ Received 11 February 2003 / Accepted 10 April 2003

\begin{abstract}
Time series of two-dimensional spectra and corresponding broad band images of solar pore regions have been obtained with the "Göttingen" Fabry-Perot Interferometer at the Vacuum Tower Telescope in Izaña, Tenerife. Line bisector shifts have been used for the computation of line-of-sight velocities in the studied regions. Additionally, a local correlation tracking (LCT) algorithm has been applied to obtain horizontal flow velocities from the time series of broad band images. Resulting velocity maps show that within the pore umbrae the flows are almost completely inhibited by the magnetic fields. This also holds for umbral dots and light bridges. On the boundaries of several pore umbrae persistent downflow channels are visible. The structure and temporal evolution of these phenomena have been studied. Finally, the formation of a protopore out of magnetic bright points has been analyzed. The obtained observational results are in good agreement with numerical models and common theoretical scenarios explaining the behaviour and formation of solar pores.
\end{abstract}

Key words. Sun: photosphere - Sun: magnetic fields - Sun: sunspots

\section{Introduction}

Solar pores are photospheric magnetic structures with intermediate sizes of several $10^{3} \mathrm{~km}$. They are not small-scale - like bright points or magnetic knots which have sizes from a few hundred kilometers down to the resolution limit of present observational data (or even below) - yet they also do not achieve the sizes of mature sunspots which are in the range of several $10^{4} \mathrm{~km}$. A characteristic feature of solar pores is that they do not fully develop a penumbra although rudimentary penumbral structures are sometimes visible around them (see e.g. Leka \& Skumanich 1998 or Sobotka et al. 1999). The magnetic field lines are found to be roughly vertical in the center of pores and they are inclined by about $40^{\circ}$ (see Keppens \& Martínez Pillet 1996) at their boundaries. Sütterlin et al. (1994) found inclination angles up to $60^{\circ}$. The field strengths are in the range between $1900 \mathrm{G}<B<2600 \mathrm{G}$ (Brants \& Zwaan 1982) and $600 \mathrm{G}<B<1700 \mathrm{G}$ (Keppens \& Martínez Pillet 1996; Sütterlin et al. 1996), respectively. Sütterlin (1998) has pointed out that the measured field strengths are strongly dependent on the formation height of the observed spectral lines and has given a vertical field gradient of $4.6 \mathrm{G} \mathrm{km}^{-1}$.

The structure of pore umbrae is not uniform, but they exhibit significant variations of continuum brightness in the range between about $30 \%$ and $120 \%$ of the mean photospheric intensity, $I_{\text {phot }}$ (Bonet et al. 1995). Similar as in the umbrae of mature sunspots pores contain a large variety of bright structures like umbral dots or light bridges. (For detailed studies of these structures see e.g. Sobotka et al. 1999 or Hirzberger et al. 2002.) These structures might be a manifestation that

\footnotetext{
* e-mail: jkh@igam.uni-graz.at
}

convective motions are not completely inhibited in the subphotospheric layers. Parker (1979) and more recently Choudhuri (1986) and Knölker \& Schüssler (1988), therefore, have suggested a pore model in which the umbra is composed of a bundle of thin flux tubes which do not completely inhibit the convective motions between them. Confirming this view, Muglach et al. (1994) have found an anticorrelation between temperature and magnetic field strength in solar pores.

From an evolutional point of view solar pores constitute a transitory state between small-scale magnetic elements and sunspots. Small scale magnetic elements grow in size by accumulating magnetic flux (see e.g. Zwaan 1985; Leka \& Skumanich 1998). When these structures achieve sizes of about $300 \mathrm{~km}$ (cf. Keller 1992) they become dark due to a reduction of the convective energy transport in the subphotospheric layers. This darkening of growing pores has been observed by e.g. Brants \& Steenbeek (1985).

The growth of pores can be maintained only if they are constantly supplied with magnetic flux from their surroundings. The large scale horizontal flow fields around pores indeed seem to be directed towards the pores (Wang \& Zirin 1992; Sobotka et al. 1999; Roudier et al. 2002). Moreover, Keppens \& Martínez Pillet (1996) have shown that the magnetic radius of pores is much larger than their visible radius.

At the very beginning of the formation of pores one sees magnetic structures (protopores or magnetic knots) which are visible as abnormally dark and broad intergranular lanes. Within these structures downward-directed vertical motions with velocities between $0.5 \mathrm{~km} \mathrm{~s}^{-1}$ and $2 \mathrm{~km} \mathrm{~s}^{-1}$ can be detected (Brants 1985; Leka \& Skumanich 1998). According to this observational result Zwaan (1985) concluded that these 
Table 1. Observing parameters corresponding to the four studied pore regions. $\alpha$ denotes the direction to disk center (see Fig. 1; counterclockwise from the $x$-axis); $\cos \theta$ is the off-center position; $\lambda_{1 / 2}$ is the $F W H M$ of the FPI; $N_{\lambda}$ and $\Delta \lambda$ give the number of spectral positions in one scan and the distance between two subsequent spectral positions; $t_{\mathrm{ex}}$ denotes the exposure times; $N_{\text {scan }}$ is the number of scans in the time series and $\Delta t$ gives the time intervals between two subsequent scans, respectively.

\begin{tabular}{cccccccccccc}
\hline \hline region & NOAA No. & date & $\alpha\left[^{\circ}\right]$ & $\cos \theta$ & line & $\lambda_{1 / 2}[\mathrm{~m} \AA]$ & $N_{\lambda}$ & $\Delta \lambda[\mathrm{m} \AA]$ & $t_{\text {ex }}[\mathrm{ms}]$ & $N_{\text {scan }}$ & $\Delta t[\mathrm{~s}]$ \\
\hline P1 & 8737 & Oct. 23, 1999 & -24 & 0.97 & Fe I 5576 & 52 & 11 & 35.25 & 20 & 19 & 70.0 \\
P2 & 8737 & Oct. 23, 1999 & -48 & 0.98 & Fe I 5576 & 52 & 11 & 35.25 & 20 & 19 & 70.0 \\
P3 & 9140 & Aug. 31, 2000 & 156 & 0.85 & Fe I 7090 & 56 & 15 & 29.78 & 20 & 49 & 47.7 \\
P4 & 9143 & Aug. 31, 2000 & 75 & 0.92 & Fe I 7090 & 56 & 15 & 29.78 & 20 & 49 & 47.7 \\
\hline
\end{tabular}

downdrafts are due to a convective collapse (see Spruit 1979) which intensifies the magnetic field strengths until the pore formation is completed. The downdrafts cease when this state is achieved. Similarly, Title et al. (1987) have found a good correlation between downflows and magnetic structures with field strengths below $650 \mathrm{G}$ and a sharp drop of the correlation for field strengths above this value. From this result the authors conclude that the downflows might surround the regions of highest field strength.

In contradiction to the previously cited results Keil et al. (1999) have found a significant correlation between magnetic flux and downflow velocities in larger pores too. Additionally, they have shown that downflow velocities peak at the visible boundaries of several pores, i.e. the strongest downflows form an annular structure around the pore umbrae. This result is in good agreement with model calculations of Deinzer et al. (1993), Knölker \& Schüssler (1988), and Steiner et al. (1998). All these theoretical models exhibit fast downward directed flow velocities just outside of magnetic flux tubes - independent of the diameter of the simulated magnetic structure, i.e. from small scale magnetic elements until pore umbrae. This result has been also confirmed by numerical models of Hurlburt \& Rucklidge (2000) who have found that even in mature sunspots the convective flows are directed downward just outside their magnetic bulks, i.e. beneath their penumbrae.

\section{Observations and data reduction}

The observations were carried out on October 23, 1999 and on August 31, 2000 with the "Göttingen" Fabry-Perot Interferometer (FPI, see Bendlin et al. 1992) which is located in the $70 \mathrm{~cm}$ Vacuum Tower Telescope (VTT) at the Observatorio del Teide, Tenerife. Time series of spectral scans across the non-magnetic Fe I $5576 \AA$ and Fe I $7090 \AA$ lines were obtained simultaneously with bursts of broad band images $(50-100 \AA$ $F W H M$ ) centered at the same wavelengths. Table 1 shows further parameters applied for the setup of the FPI.

The basic data reduction is the same as described in Hirzberger et al. (2001) and Hirzberger \& Kneer (2001), i.e. after correcting for dark offsets, flat fields, and global image motions the broad band data have been reconstructed using speckle interferometric techniques (see de Boer 1993) and the narrow band data have been reconstructed for optical transfer functions obtained from a division (in the Fourier domain) of the raw broad band data by the speckle reconstructed broad band data (for details see Krieg et al. 1999). In a subsequent step Doppler maps for each spectral scan of the time series have been obtained from the line bisectors computed in each pixel of the field of view. For the present study Doppler maps were computed only at line depths $I_{\text {core }}+\left(I_{\text {cont }}-I_{\text {core }}\right) / 2$. Here, $I_{\text {core }}$ and $I_{\text {cont }}$ denote the local line core intensities and continuum intensities, respectively. These line depths correspond to photospheric heights between approximately $100 \mathrm{~km}$ and $130 \mathrm{~km}$ above the continuum level (see Altrock et al. 1975; Hirzberger et al. 2001). Absolute values of the Doppler velocities have been defined by setting the mean velocity in granular subfields of the images equal to zero. In this connection a small error has to be taken into account since these subfields are rather small and different relative areas between upflows and downflows must be considered. However, the mean velocities in these subfields are close to the mean velocities in the pore umbrae (cf. Sect. 3.1), i.e. the differences are smaller than the expected error level of the velocity maps. Consequently, the error due to this "convective blueshift" should be negligible.

Thereafter, time series of broad band images have been destretched in order to remove residual image distortions. This has been carried out applying a local correlation tracking (LCT) code developed by Yi \& Molowny Horas (1992). The obtained image distortion maps have been used for destretching the corresponding Doppler maps too. The last step of the data reduction procedure was to filter out acoustic waves by the application of a subsonic filter with cut-off phase speeds of $5 \mathrm{~km} \mathrm{~s}^{-1}$. The achieved spatial resolution of the reconstructed broad band images is approximately $0 . ! 3$ and that of the Doppler maps about 0.4 (see also Hirzberger et al. 2001). The residual rms noise in the the Doppler maps is depending on the amount of light in the narrow band channel of the FPI system, i.e. on the photospheric intensity. Its values are in the range of about $\pm 50 \mathrm{~m} \mathrm{~s}^{-1}$ in granular regions and approximately $\pm 100 \mathrm{~m} \mathrm{~s}^{-1}$ in the pore umbrae.

Figure 1 shows examples of reconstructed broad band images of the four pore regions analyzed in the present study. Regions $\mathrm{P} 1$ and $\mathrm{P} 2$ are parts of a small active region (NOAA 8737, Zürich classification $\mathrm{C}$ ), whereas regions $\mathrm{P} 3$ and $\mathrm{P} 4$ are located in the outer regions of large bipolar spot groups (NOAA 9140 and NOAA 9143, Zürich classification E and D).

\section{Results}

\subsection{Time averaged line-of-sight velocities}

In Fig. 2 time averaged line-of-sight velocities, $\left\langle v_{\text {LOS }}\right\rangle$, of the four pore regions are displayed. The averaging process has 

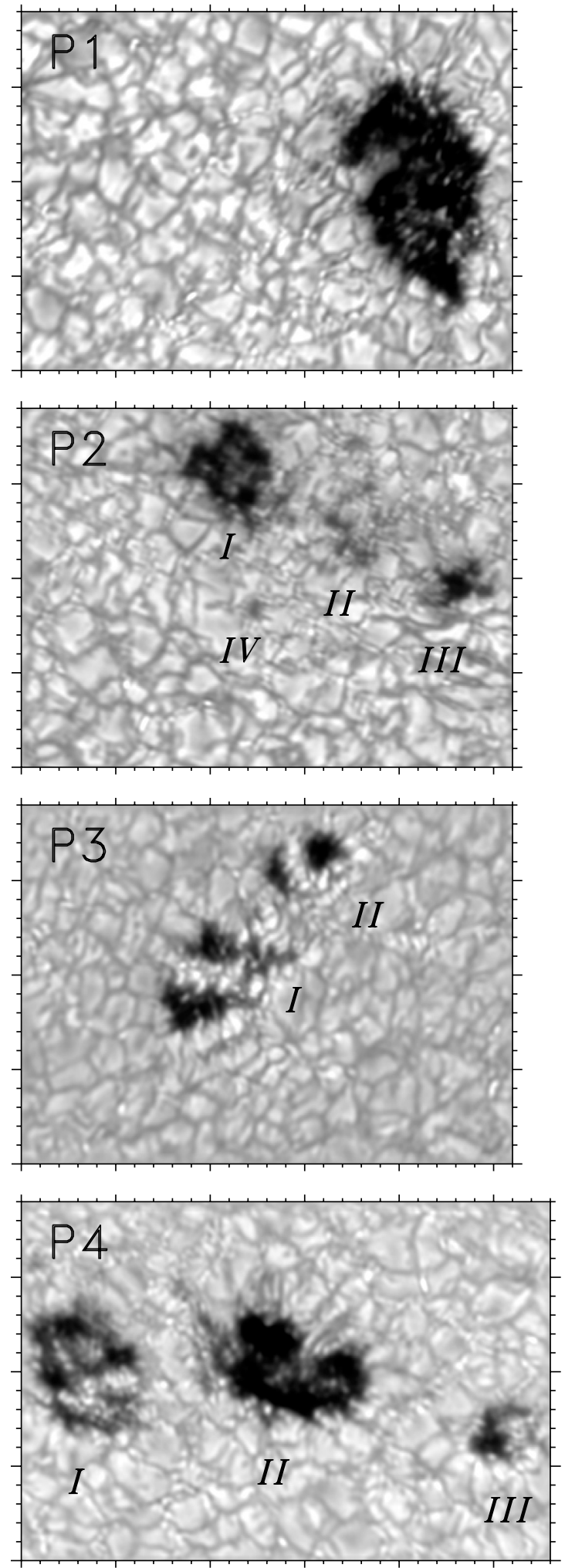

Fig. 1. Broad band images of the analyzed pore regions. For details see text and Table 1. Tickmarks are (here and in Figs. 2, 3, 5, 7, and 9) at distances of $1^{\prime \prime}$.

been carried out including all Doppler maps of the corresponding time series, that is over $21 \mathrm{~min}$ for $\mathrm{P} 1$ and $\mathrm{P} 2$ and over 38 min for $\mathrm{P} 3$ and $\mathrm{P} 4$, respectively. The overplotted contour lines denote $75 \%$ of the mean photospheric intensity of the
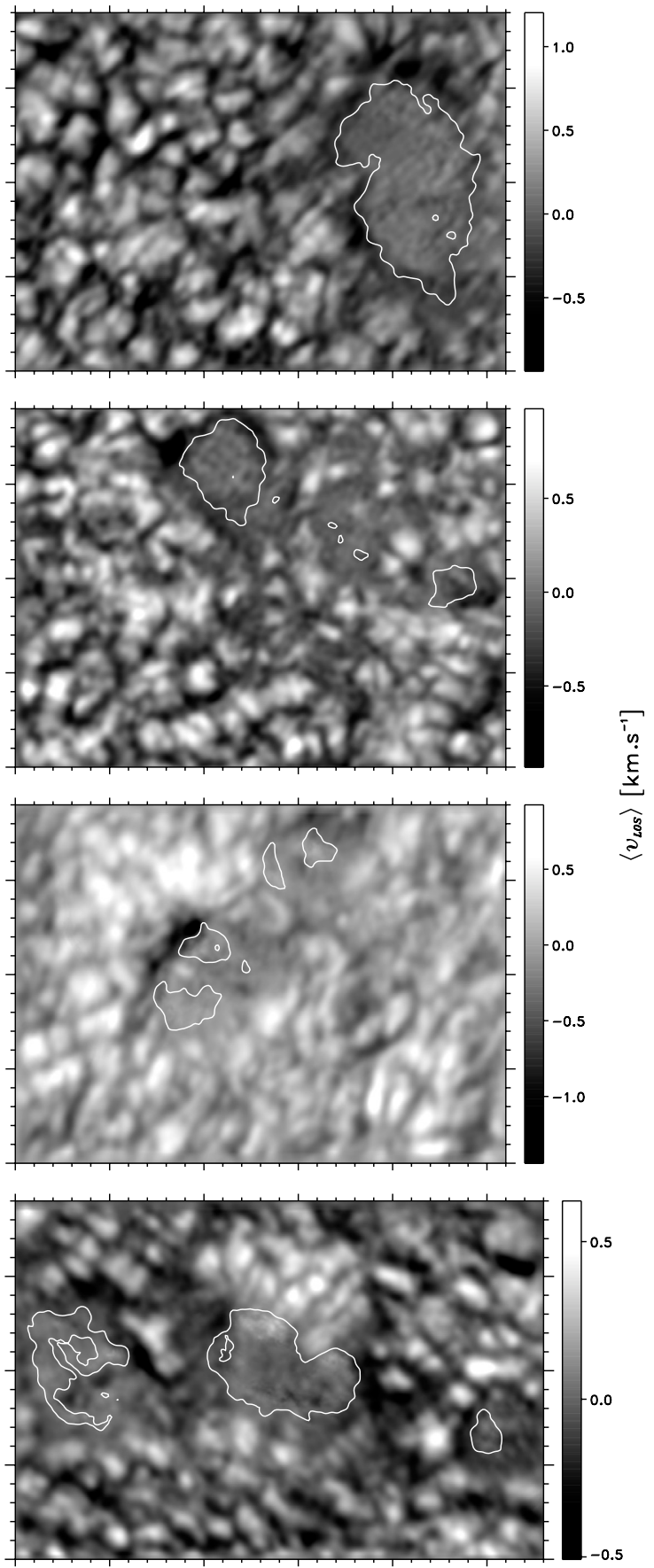

Fig. 2. Time averaged Doppler maps of the analyzed pore regions. Bright regions (positive values) denote upflows. Contour lines are at $\left\langle I_{\mathrm{BB}}\right\rangle=0.75$.

time averaged broad band images $\left(\left\langle I_{\mathrm{BB}}\right\rangle=0.75\right)$. The most significant property visible in Fig. 2 is that the granular flow pattern is not smeared out, i.e. the Doppler maps are dominated by long-lived or recurring structures. Within the pore umbrae the averaged vertical velocities are generally very small although different in the four studied regions. For example, the variation of $\left\langle v_{\mathrm{LOS}}\right\rangle$ within the large pore in $\mathrm{P} 1$ and pore $\mathrm{P} 2 I$ is not higher than the expected noise level. The mean $\left\langle v_{\mathrm{LOS}}\right\rangle$ in these pore umbrae is close to the mean $v_{\text {LOS }}$ in quiet granular subfields, which has been set to zero. The same is valid for P2II. 
This region contains several small and fast evolving dark features. $\left\langle v_{\mathrm{LOS}}\right\rangle$ is zero throughout this entire region even far beyond the plotted contours of the averaged intensity.

Just outside the umbrae of several pores rather fast and persisting downflows can be detected. This can be seen best on the upper boundary of P2I or just left to pore P3I. In this latter region the minimum velocity amounts to $\left\langle v_{\text {LOS }}\right\rangle=-1.4 \mathrm{~km} \mathrm{~s}^{-1}$ which is surprisingly fast for this $38 \mathrm{~min}$ average. Around P2I the local downflow centers achieve velocities between $\left\langle v_{\text {LOS }}\right\rangle=$ $-0.4 \mathrm{~km} \mathrm{~s}^{-1}$ and $\left\langle v_{\mathrm{LOS}}\right\rangle=-0.9 \mathrm{~km} \mathrm{~s}^{-1}$. Also outside the lefthand boundary of the large pore in $\mathrm{P} 1$ persistent downflows can be detected. Here the local downflow centers show velocities between $\left\langle v_{\mathrm{LOS}}\right\rangle=-0.4 \mathrm{~km} \mathrm{~s}^{-1}$ and $\left\langle v_{\mathrm{LOS}}\right\rangle=-0.7 \mathrm{~km} \mathrm{~s}^{-1}$. (The different velocity scales of the grey levels in the four panels of Fig. 2 have to be noticed.)

The averaged Doppler map of region P4 shows some more interesting features. Similar as in the other studied regions close to the upper right boundary of P4I an approximately 8 " long downflow channel can be detected. Moreover, at the righthand side of P4II a persistent downflow region is visible. The flow centers achieve velocities between about $\left\langle v_{\mathrm{LOS}}\right\rangle=$ $-0.3 \mathrm{~km} \mathrm{~s}^{-1}$ and $\left\langle v_{\mathrm{LOS}}\right\rangle=-0.5 \mathrm{~km} \mathrm{~s}^{-1}$.

A large fraction of the area of $\mathrm{P} 4 \mathrm{I}$ is dominated by rather bright structures such as umbral dots and granular light bridges (see Sobotka et al. 1994 and Sobotka 1997 for nomenclature). In this pore also the Doppler map shows some structures. Two downflow regions with velocities of about $\left\langle v_{\mathrm{LOS}}\right\rangle \approx$ $-0.2 \mathrm{~km} \mathrm{~s}^{-1}$ are visible. It is interesting that these two regions are co-spatial with the brightest features of the umbra (see Fig. 1), i.e. they are located outside the contour line in Fig. 2 which separates the light bridges from the rest of the umbra. In contrast, in the strong light bridge which divides the two umbral regions of P3I a weak upflow region can be detected.

A further conspicuous phenomenon visible in region $\mathrm{P} 4$ is a large area with positive $\left\langle v_{\mathrm{LOS}}\right\rangle$ above the large pore $\mathrm{P} 4 I I$. This flow field possibly can be attributed to an Evershed-like outflow from the pore. This can be concluded since this flow appears on the center-side boundary of the pore (cf. Table 1) and since the white light images of this region (see Fig. 1) show some filamentary structures which might be parts of a rudimentary penumbra. An evident detail concerning this Evershed-like flow is that it seems to have a well-defined outer boundary. On this boundary $\left\langle v_{\mathrm{LOS}}\right\rangle$ changes its sign, which can be explained by a change of the direction of the flows from horizontal outward to vertical downward. This would be in good agreement with results from sunspots containing a fully developed penumbra (see Hirzberger \& Kneer 2001; Westendorp Plaza et al. 2001) and with theoretical models of e.g. Montesinos \& Thomas (1997).

\subsection{Mean horizontal flow fields}

Figure 3 shows mean horizontal flow fields of the four studied pore regions. These flow fields have been computed applying an LCT algorithm (see Yi \& Molowny Horas 1992) with a Gaussian window function of 1"' $2 F W H M$. The resulting flow velocities, $\left\langle v_{\mathrm{H}}\right\rangle$, show a smooth pattern in regions $\mathrm{P} 3$ and $\mathrm{P} 4$
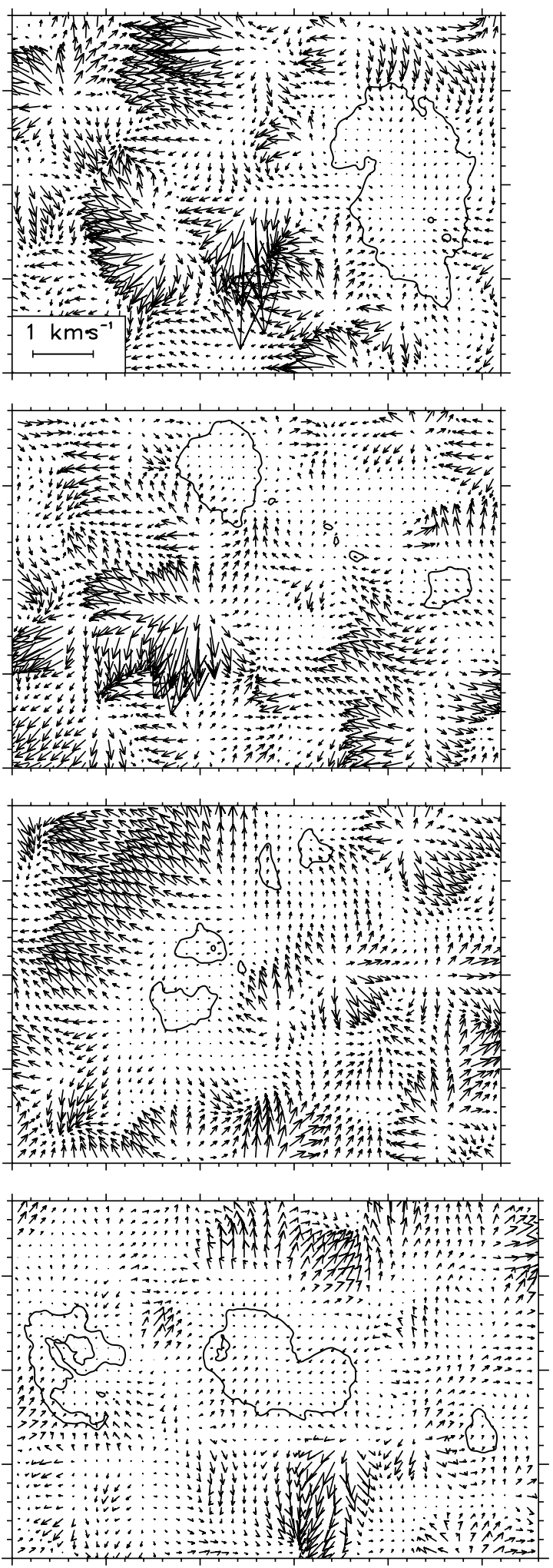

Fig. 3. Time averaged horizontal flow fields obtained with LCT methods. Contour lines are the same as in Fig. 2.

and are considerably more structured in regions P1 and P2. This difference appears because of the different lengths of the time series (cf. Table 1). The maximum horizontal velocities are $\left\langle v_{\mathrm{H}}\right\rangle_{\max }=3.8 \mathrm{~km} \mathrm{~s}^{-1}$ in region P1 and $\left\langle v_{\mathrm{H}}\right\rangle_{\max }=1.2 \mathrm{~km} \mathrm{~s}^{-1}$ 

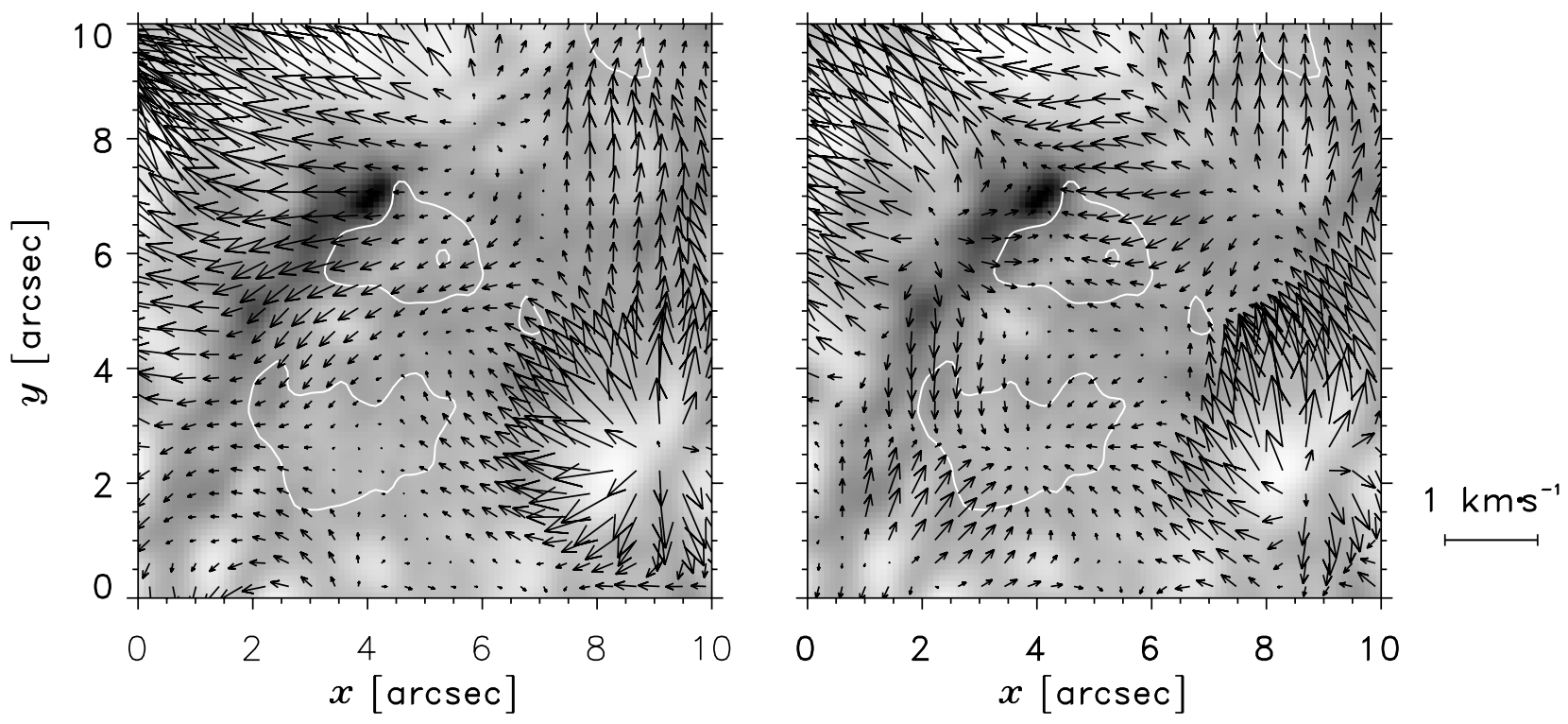

Fig. 4. Enlargements of the averaged Doppler map around P3I overplotted by horizontal flow fields calculated with an LCT algorithm using a Gaussian window function of 1'. 2 FWHM. The flow field of the left panel has been derived from the first 25 images of the time series, the one in the right panel from the last 24 images of the time series. Contour lines are the same as in Fig. 2.

in region P3, respectively. Although the studied time series are in general rather short the resulting flow fields represent quite well the visual impression gained from movies of the four time series, i.e. the LCT algorithm produces realistic outputs. For example, the conspicuous divergence center below P2I is produced by a cascade of exploding granules.

Within and around the pore umbrae the horizontal flow velocities are very low. This is in good agreement with results of Keil et al. (1999) who have found a reduction of horizontal flow velocity with increasing magnetic flux density. The highest velocities surround the umbrae at distances between $5^{\prime \prime}-10^{\prime \prime}$. This agrees only partially with results presented by Roudier et al. (2002). These authors have found divergence centers, i.e. "rosetta"-like structures, which surround the pore umbrae and do also drive radial inflows into the pores. Divergence maps calculated from the horizontal flow fields of the present data (not shown) confirm the impression that the pores are surrounded by cellular structures containing positive divergence values. Nevertheless, in contrast to the results obtained by Roudier et al. (2002) the flow fields are rather asymmetric, i.e. the absolute values of $\left\langle v_{\mathrm{H}}\right\rangle$ are much smaller for flows from the divergence centers towards the pores than into opposite directions. This result is the more interesting since the persistent downflow channels which surround many of the pores studied in the present paper need sources for fulfilling mass continuity and, hence, horizontal inflows would be a good explanation for their appearance. Horizontal inflows into the pores can be seen on movies of the studied time series but they do not completely surround the pores as found by Roudier et al. (2002) or Sobotka et al. (1999). The flow maps displayed in Fig. 3 confirm this impression. These inflows occur only on a few parts of the umbral boundaries, e.g. on the lower left boundary of P2I or on the upper boundary of the pore in P1. Both regions do not develop really continuous downflows (cf. Fig. 5) although $\left\langle v_{\mathrm{LOS}}\right\rangle$ is slightly below zero in that regions. In the regions of the most conspicuous persistent downflow channels (e.g. at the upper boundary of P2I or at the left boundary of P3I) the horizontal velocities are close to zero, i.e. horizontal inflows do not seem to be the sources for these phenomena.

The strongest persistent downflow channel is visible left of P3I. In Fig. 4 enlargements of horizontal flow fields in a small region around this pore are displayed. These two flow fields have been calculated once for the first 25 images of the time series and once for the last 24 images of the time series, respectively. They do not deviate very much from each other and they are also similar to the flow chart displayed in Fig. 3. This is a confirmation that the flow field does not change significantly along the time series and another corroboration that the applied LCT algorithm produces realistic outputs. The major difference between the flow charts displayed in Figs. 3 and 4 are the somewhat higher velocities which are expected when applying a shorter averaging time interval. Again there is no significant inflow into the pore umbra visible at the position of the persistent downflow channel. On the opposite side of the pore, i.e. at approximately $x=8 . \prime 5, y=2 . .5$, a strong divergence center drives a flow towards the pore but the velocities cease almost completely at the umbral boundary. The right panel of Fig. 4 shows a horizontal inflow into the lower part of P3I. This inflow may correspond to those found by Roudier et al. (2002), however, the maximum velocity, $\left\langle v_{\mathrm{H}}\right\rangle=275 \mathrm{~m} \mathrm{~s}^{-1}$, is located at the lower left boundary of the pore where no downflow channel is visible.

\subsection{Time evolution of downflow channels}

In the previous sections it has been stated that many of the studied pores are - at least partially - surrounded by persistent downflow channels. In Fig. 5 six snapshots of the time evolution of P2I are displayed. Its surrounding downflow patches are permanently changing positions, sizes and shapes. To show 


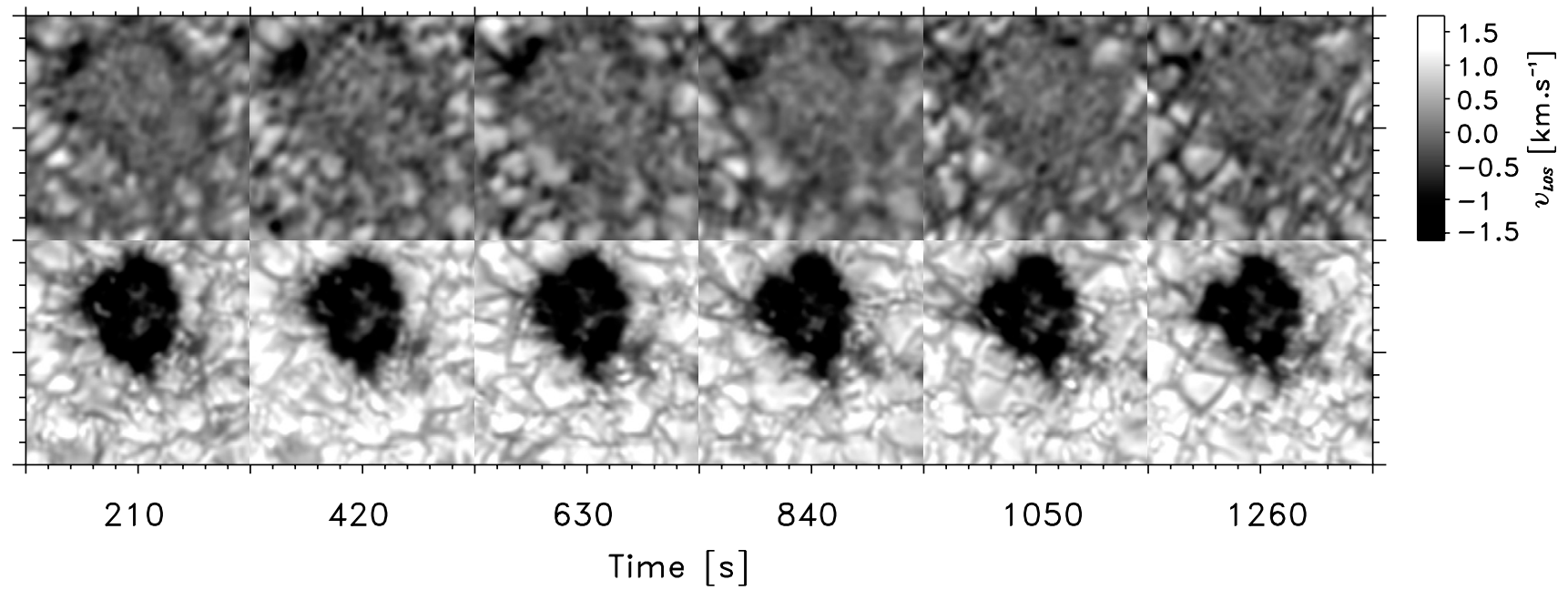

Fig. 5. Six snapshots of the evolution of $\mathrm{P} 2 I$. The lower panels show the broad band intensities, $I_{\mathrm{BB}}$, in the upper panels the corresponding line-of-sight velocities, $v_{\mathrm{LOS}}$, are displayed.

this in a more quantitative manner in Fig. 6a the time evolution of the area (around P2I) where $v_{\mathrm{LOS}}<-0.5 \mathrm{~km} \mathrm{~s}^{-1}$ is displayed. Overplotted is the apparent time evolution of the average $v_{\text {LOS }}$ in these areas. The two curves run almost parallel, i.e. the velocities have the higher amplitudes the larger the downflow channel area is.

In Fig. $6 \mathrm{~b}$ the temporal evolution of the downflow channel close to P3I is displayed. Plotted are the temporal variations of $I_{\mathrm{BB}}$ and $v_{\mathrm{LOS}}$ averaged in a $9 \times 9$ pixel area centered at the minimum $\left\langle v_{\mathrm{LOS}}\right\rangle$. Again a significant temporal variation of the downflow velocity is visible. Also the broad band intensity exhibits a strong variation although this downflow channel is located in a rather dark region with intensities $0.79<I_{\mathrm{BB}}<0.88$. The variations of the two curves displayed in Fig. $6 \mathrm{~b}$ do not seem to run exactly in phase. In the first 12 min both parameters run parallel but then the intensity is already increasing again whereas the velocity minimum appears several minutes later.

\subsection{Intensity structure of the downflow channels}

As already mentioned above, the downflow channels seem to be situated in rather dark regions along umbral boundaries. A more significant confirmation of this statement will be given. Thereto the time averaged broad band intensity, $\left\langle I_{\mathrm{BB}}\right\rangle$, of the pore region $\mathrm{P} 2 I$ has been computed and a segmentation has been carried out by considering only areas belonging to lineof-sight velocities $\left\langle v_{\mathrm{LOS}}\right\rangle<-0.2 \mathrm{~km} \mathrm{~s}^{-1}$ and time averaged broad band intensities $0.8<\left\langle I_{\mathrm{BB}}\right\rangle<0.9$. The result is displayed in Fig. 7 where the contours of the segmented area are overplotted to the time averaged Doppler map. In Fig. 8a a velocity-intensity scatter plot $\left(v_{\mathrm{LOS}}\right.$ vs. $\left.I_{\mathrm{BB}}\right)$ of the segmented area exhibited in Fig. 7 and projected to all images of the time series is shown. This scatter plot exhibits no conspicuous mutual dependence of the two parameters although two main components of the distribution can be distinguished. These two different types of pixels can be interpreted if the two scatter plots shown in Figs. 8b and $\mathrm{c}$ are viewed. Figure 8b shows
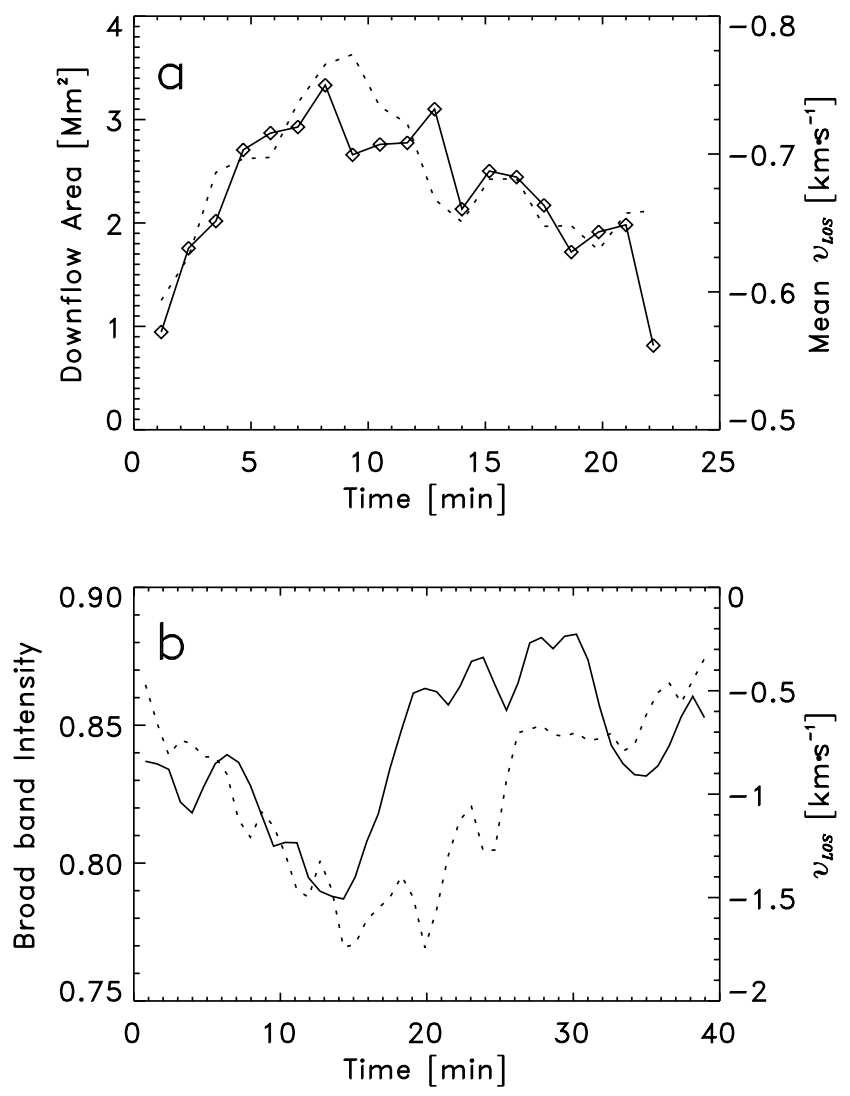

Fig. 6. a) Temporal evolution of the area (around P2I, see Fig. 5) where $v_{\mathrm{LOS}}<-0.5 \mathrm{~km} \mathrm{~s}^{-1}$ (solid line) and of the mean $v_{\mathrm{LOS}}$ within this area (dotted line); b) evolution of $I_{\mathrm{BB}}$ (solid line) and $v_{\mathrm{LOS}}$ (dotted line) averaged in a $9 \times 9$ pixel area around the position of the minimum velocity in the time averaged Doppler map of P3 (see Fig. 2).

the intensity-velocity distribution of the pixels in a rather quiet granular field located in the lower left corner of the region P2. Here a clear linear dependence of the line-of-sight velocity on the broad band intensity can be seen. The linear fit gives a slope of $\Delta v_{\mathrm{LOS}} / \Delta I_{\mathrm{BB}}=6.04 \mathrm{~km} \mathrm{~s}^{-1}$. The scatter plot shown 


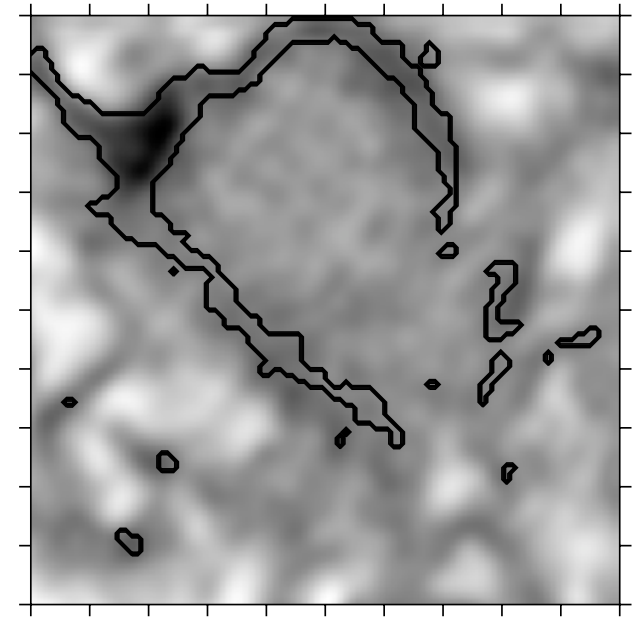

Fig. 7. Time averaged Doppler map around P $2 I$ overplotted by the contours of the segmented area considered in Fig. 8a.

in Fig. 8c includes all pixels belonging to the time averaged umbral boundary, i.e. to the boundary of the region where $\left\langle I_{\mathrm{BB}}\right\rangle>0.8$ projected to all images of the time series. This plot shows an almost uncorrelated cloud. It can be fitted by a regression line with a slope of $\Delta v_{\mathrm{LOS}} / \Delta I_{\mathrm{BB}}=1.09 \mathrm{~km} \mathrm{~s}^{-1}$ although the correlation of the fit is with $c=0.2$ rather low.

The linear fits obtained from the two scatter plots in Figs. $8 \mathrm{~b}$ and $\mathrm{c}$ are a good representation of the two components of pixels in the downflow channel (see the overplotted lines in Fig. 8a). Hence, it can be concluded that the downflow channels might not be much different from quiet intergranular lanes. The second component of pixels visible in Fig. 8a seem to belong to the umbral boundary, i.e. they are relative dark but do not exhibit fast line-of-sight velocities.

\subsection{Formation of a protopore}

In the time series of region $\mathrm{P} 2$ the formation of a magnetic knot or a protopore can be traced (P2IV, see Fig. 1). This event is displayed in Fig. 9. The broad band images (lower panels) show that the protopore forms in an abnormal granulation region. Before the dark protopore appears (at $t<560 \mathrm{~s}$ ) at its position several bright structures with sizes of about $0 . " 3 \mathrm{di}-$ ameter are visible. They are probably magnetic bright points since in the corresponding line center images (not shown) local brightenings can be recognized in that region. The corresponding Doppler maps (middle and upper panels of Fig. 9) show several localized downflows situated at that position. At $t=560 \mathrm{~s}$ for the first time a tiny structure with a broad band intensity below $I_{\mathrm{BB}}=0.85$ is visible. This structure is surrounded by four downflows with sizes of about 0 !" 5 . As the dark region increases also the downflow areas grow until they form a vast downflow region with velocities below $-1 \mathrm{~km} \mathrm{~s}^{-1}$ which partially overlaps the protopore.

The bright points are disappearing as the protopore grows. The one marked by the white arrow at $t=560 \mathrm{~s}$ is an example on which the disappearance can be traced. It shrinks until at $t=770 \mathrm{~s}$ it seems to be connected with the above granule but at $t=910 \mathrm{~s}$ it is not visible anymore. $v_{\mathrm{LOS}}$ in this structure is
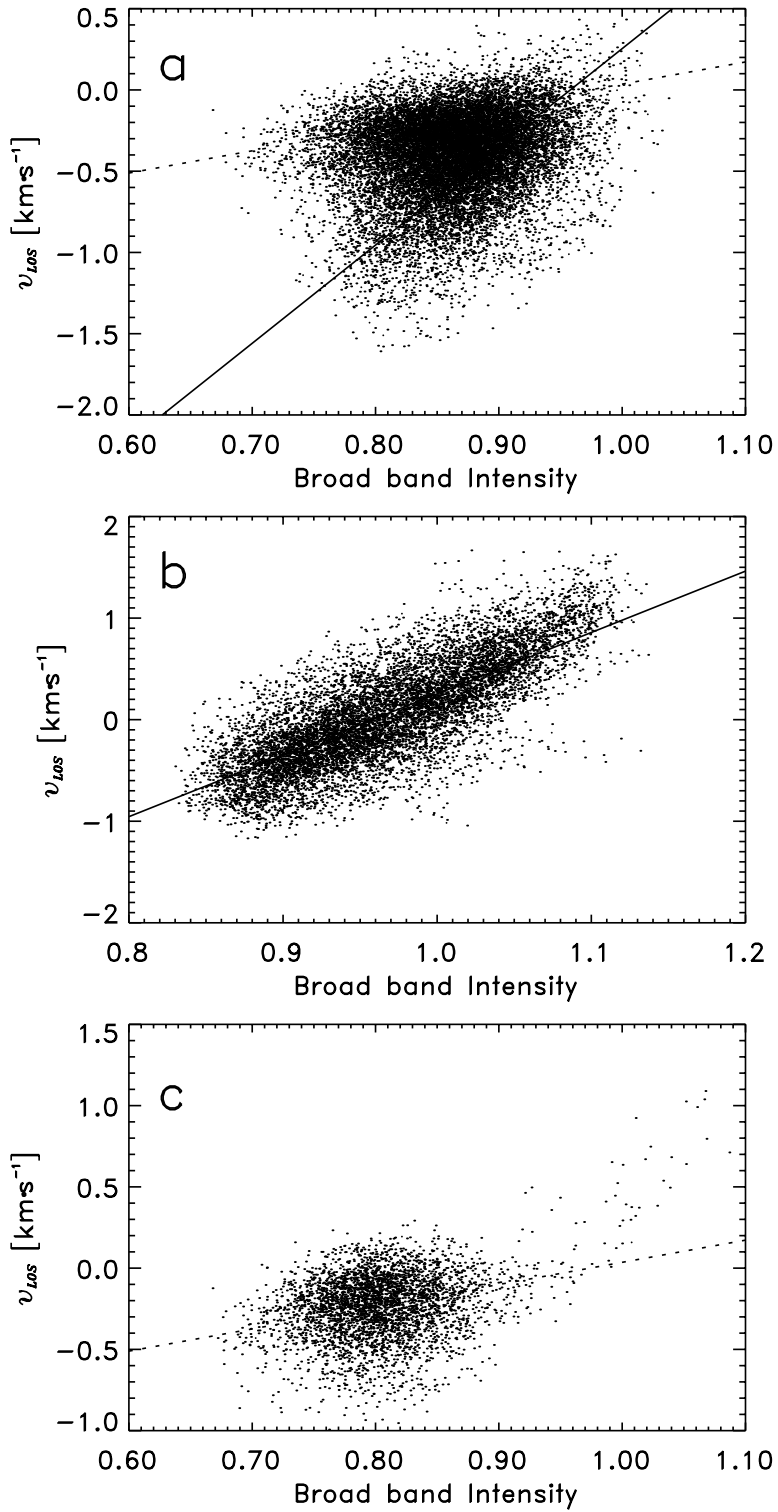

Fig. 8. Velocity vs. intensity scatter plots of a) the downflow channel surrounding P2I (segmented area shown in Fig. 7); b) a quiet granular field in the lower left corner of $\mathrm{P} 2$; c) the umbral boundary of $\mathrm{P} 2 I$ (see text). The solid and the dotted lines represent linear fits to the scatter plots in panel b) and c) and have been overplotted to panel a).

close to zero as long as it is visible. Later on (at $t \geq 910 \mathrm{~s}$ ) a downflow can be recognized at the position where this structure has disappeared. This result agrees well with the suggestion of Zwaan (1985) that growing pores are accumulating magnetic flux by the convective collapse mechanism.

Another conspicuous event visible in Fig. 9 is the expansion of a granule just left to the growing protopore. This granule develops a broad intergranular lane between itself and the protopore. From $t=700 \mathrm{~s}$ on, this intergranular lane is connected with the downflow region belonging to the protopore, so that the two downflow regions are not distinguishable anymore. An interesting question now is whether the granule expansion somehow triggers the pore formation. Muller \& Roudier (1992) have suggested that the compression of magnetic fields between expanding granules represents a possible formation 

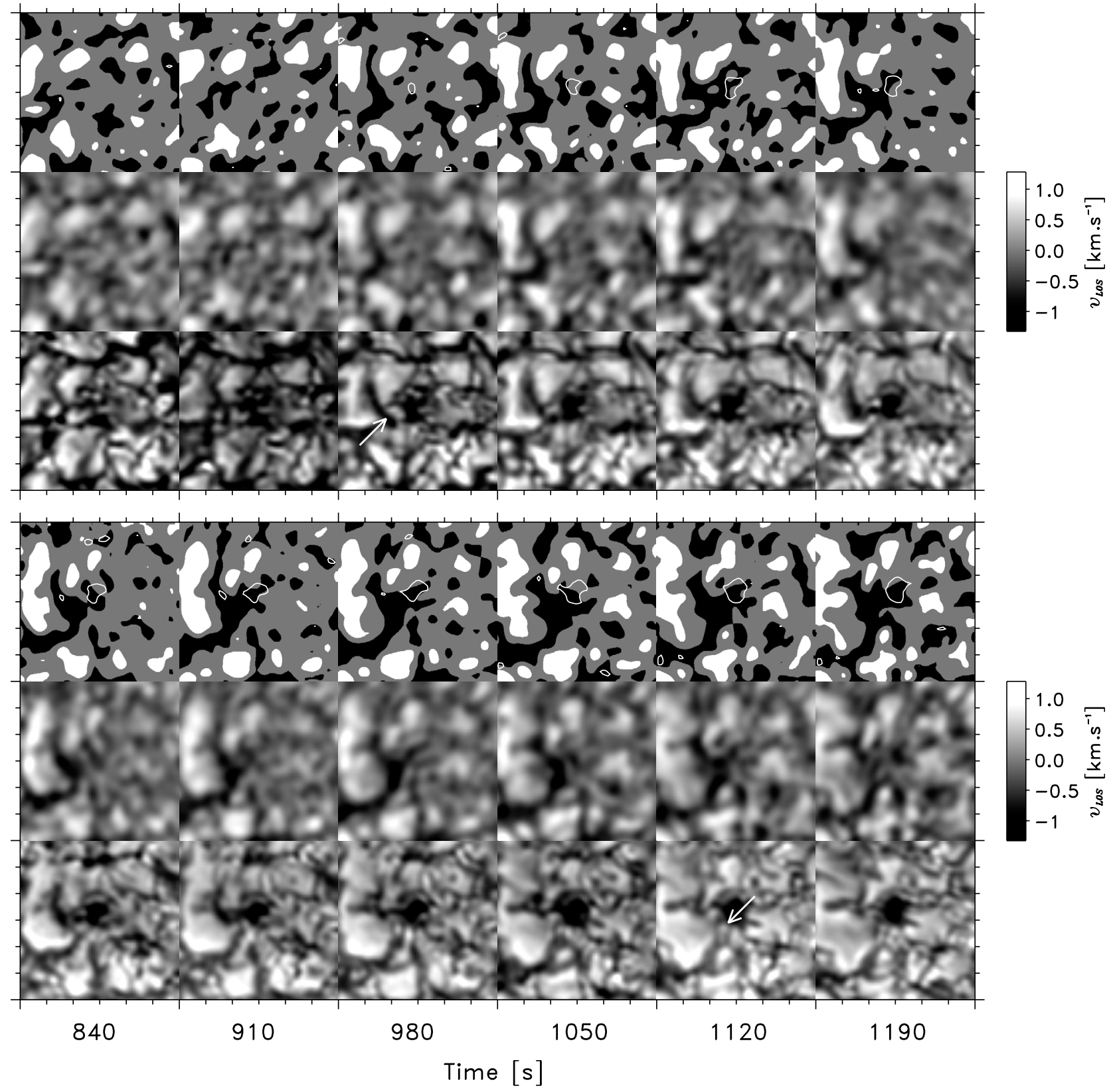

Fig. 9. Twelve snapshots of the evolution of region P2IV, i.e. of the formation of a protopore. Lower panels show broad band images; in the middle panels the corresponding Doppler maps are displayed; upper panels show segmented Doppler maps with loos $\geq 0.3 \mathrm{~km} \mathrm{~s}{ }^{-1}$ as white areas and with $v_{\mathrm{LOS}} \leq-0.3 \mathrm{~km} \mathrm{~s}^{-1}$ as black areas, respectively. White contours denote broad band intensities $I_{\mathrm{BB}}=0.85$.

mechanism for bright points. Sobotka et al. (1999) and Roudier et al. (2002) have found "rosetta"-shaped divergent horizontal flows located around pores. Hence, growing pores might be supplied with magnetic flux by the expansion of granules. The expanding granule in Fig. 9 consists of two parts. The upper part is slowly expanding and another small granule between the expanding one and the protopore is slowly decaying. The lower part is expanding faster and the growth proceeds towards the protopore. While this lower part of the granule grows, several bright points are appearing between the granule and the protopore. They are best visible at $t=1120 \mathrm{~s}$. The corresponding line-of-sight velocities are different in these bright points. Whereas in most of them $v_{\mathrm{LOS}}$ is close to zero the one marked with the arrow (at $t=1120 \mathrm{~s}$ ) has $v_{\mathrm{LOS}}=-0.8 \mathrm{~km} \mathrm{~s}^{-1}$. Although the size of this bright point is close or even below the resolution limit of the Doppler maps and, moreover, some scattered light from the broad downflow region around it has to be considered it is, however, possible that here another convective collapse is visible. The last snapshot displayed in Fig. 9 $(t=1190 \mathrm{~s})$ shows that the expanding granule starts to decay; this is best visible in the segmentation of the Doppler map. Additionally, the intensity of the bright points is reduced in this image. Thus, the intensity in this region is generally decreasing whereas the downflow region is continuously expanding. 
Unfortunately the time series is not longer so that it remains unclear whether the protopore grows further by absorbing the bright points.

\section{Discussion and conclusions}

Based on high spatial resolution two-dimensional spectroscopic data the flow fields in and around solar pore regions have been analyzed. Within the pore umbrae the detected line-ofsight velocities are mainly close to zero, i.e. the plasma motions are inhibited for the major part by the magnetic field structure. Some of the analyzed pores contain a rich internal structure of umbral dots and light bridges. Even within these structures the detected $v_{\text {LOS }}$ hardly exceed the noise level of the data. Only two exceptions have been found: In the time-averaged flow map of (i) a strong light bridge which divides the umbra of pore P3I an upward velocity of approximately $0.2 \mathrm{~km} \mathrm{~s}^{-1}$ is visible, and (ii) within the brightest parts of a granular light bridge in P4I downward directed flows with velocities of about $\left\langle v_{\mathrm{LOS}}\right\rangle=-0.2 \mathrm{~km} \mathrm{~s}^{-1}$ can be found. It can be concluded from this result that also within these structures convective motions are almost totally inhibited. This result disagrees with that of e.g. Kneer (1996), Sobotka et al. (1994), or Rimmele (1997) who have found clear indications for blueshifted line profiles in umbral dots and bright grains of a strong light bridge, respectively. Numerical models of Degenhardt \& Lites (1993) also predict upward motions in bright umbral features although the simulated flow velocities decrease exponentially with height. Schmidt \& Balthasar (1997) have found that flow velocities in umbral dots must be smaller than $25 \mathrm{~m} \mathrm{~s}^{-1}$. Lites et al. (1991) also did not detect significant vertical flow velocities in umbral brightenings but they conclude that convective motions might be present below the visible surface. This discrepancy between the above cited observational results is most likely due to the different formation heights of the velocity signals shown by the different authors. Moreover, the velocity signals obtained in the present study are formed at photospheric layers where the expected convective flow velocities are below the noise level of the present data.

The most striking features in the Doppler maps are downflow channels located at the umbral boundaries of the pores. This result agrees well with observations presented by Keil et al. (1999) who also have observed redshifts in pore umbrae. In their results the maximum downflow velocities appear at the boundaries of the pores which were interpreted as annular downflow structures surrounding the analyzed pores. Contradictory to the results of Keil et al. (1999) the spatially highly resolved Doppler maps in the present paper show that the downflow channels surrounding the pores are not closed rings but their shapes are strongly changing in time. Velocity vs. intensity scatter plots show that the maximum velocities in these downflow channels occur on intergranular-like dark lanes around the pores, i.e. the dynamics of these downflow channels must be somehow related to the convective flows around the pores. Results from photometric studies of solar pores (Sobotka et al. 1999; Roudier et al. 2002) show that the horizontal flow field around solar pores is dominated by "rosetta"-like divergence centers which drive horizontal motions towards and across the umbral boundaries. The horizontal flow fields obtained from the present data show also penetrating flows at some parts of the umbral boundaries but they seem to avoid the downflow channels, i.e. at the positions of the downflow channels the horizontal flow velocities are rather small.

In the past decades several numerical models have been developed in order to simulate the behaviour of magnetic flux tubes in the solar photosphere. Independently on the diameter of the flux tubes - they are ranging from about $200 \mathrm{~km}$ (Steiner etal. 1998) via pores with about 500-1000 km (Deinzer et al. 1993; Knölker \& Schüssler 1988) until mature sunspots (Hurlburt \& Rucklidge 2000) - the models concurrently predict fast downward motions at the boundaries of the simulated structures. These numerical models also predict that the horizontal flows within the flux tubes are rather small, i.e. penetrating horizontal motions across the downflow channels are not expected. On the other hand the growth of pores is attributed to an accumulation of magnetic flux due to the convective collapse scenario (Zwaan 1985; Leka \& Skumanich 1998). Hence, a convective transport of magnetic flux towards and maybe into solar pores has to be expected.

Several authors (e.g. Wang \& Zirin 1992; Sobotka et al. 1999) have clearly shown that bright structures are able to move from outside into pore umbrae forming umbral dots. Some of these penetrating bright structures are also visible in the present time series but they mainly appear in regions where no downflow channels are located and they correspond quite well with those parts of the umbral boundaries where the computed horizontal flow velocities show an inward motion. The appearance of bright umbral structures is thought to be an indication for the decay of a pore or a sunspot (see e.g. Vázquez 1973) since they are expected to represent convective features. Hence, the penetration of bright structures can be rather attributed to an erosion of the sunspot (see Petrovay \& Moreno Insertis 1992) than to an accumulation of flux due to a convective collapse. Such a convective collapse also would require fast downward motions which definitively are not visible at the regions of penetrative horizontal flows.

The convective collapse scenario (see Spruit 1979) instead represents a suitable explanation for the formation of protopores or magnetic knots. The one studied in the present paper (see Fig. 9) forms in an abnormal granulation region out of several tiny bright structures which probably are magnetic bright points. Simultaneously with the dark protopore a fast downflow develops and while the protopore grows some more bright structures are moving towards it. In close proximity to the protopore a large expanding granule appears which is maybe responsible for the supply of magnetic flux to the growing protopore. Another explanation for the formation of a protopore out of separate bright magnetic points considers attracting vortex rings and has been proposed by Parker (1992). According to this model downdraft vortex rings, located below the surface layer and encircling individual flux tubes may produce an attracting hydrodynamic force which overcomes magnetic stresses that keep flux tubes apart. Furthermore, Parker (1992) conjectures that, after the flux tubes are bundled to pores or sunspots, the individual vortex rings may merge to form 
large downdraft vortex rings surrounding the entire magnetic structure. This conjecture, thus, represents a suitable explanation for the occurrence of downflow channels detected outside of several pores in the present data.

One of the larger pores (P4II) studied in the present paper shows a rudimentary penumbra. This pore covers an area of approximately $8^{\prime \prime} \times 4^{\prime \prime}$ and is therefore much smaller than e.g. PI which covers about $12^{\prime \prime} \times 6^{\prime \prime}$ and shows no sign to develop a penumbral structure. This overlap of sizes of sunspots and pores is in good agreement with former observations, reviewed e.g. in Bray \& Loughhead (1964) or Zwaan (1992). Numerical models of Rucklidge et al. (1995) also show a bifurcation of solutions (cellular vs. filamentary convection) if the magnetic flux is varied. Leka \& Skumanich (1998) have found a threshold of $(1-1.5) \times 10^{20} \mathrm{Mx}$ above which a pore can develop penumbral structures. The rudimentary penumbra in (P4II) manifests itself rather in the Doppler maps than in the white light images. Only a few filaments can be detected in white light. The Doppler maps show an overall filamentary structure, however, the individual filaments are composed of several knots. This could mean that maybe granular convection is overlaid by a filamentary Evershed flow. Since the contributions to the Doppler maps stem from a broad height interval (see Hirzberger et al. 2001) this interpretation might be valid although speculative. However, this interpretation would be in good agreement with numerical models of Hurlburt \& Rucklidge (2000). These models show that the inclination of the penumbral field lines is dependent on the magnetic flux content, so that possibly around the smallest sunspots both convective motions and the Evershed flow are observable.

Acknowledgements. The author is grateful to M. Sobotka for valuable comments on the manuscript. The Vacuum Tower Telescope is operated by the Kiepenheuer-Institut für Sonnenphysik in Freiburg (Germany) at the Spanish Observatorio del Teide of the Instituto de Astrofísica de Canarias in Tenerife. Financial support by the Austrian Fonds zur Förderung der wissenschaftlichen Forschung (Erwin-Schrödinger-Rückkehrprogramm No. R11) is gratefully acknowledged. The author thanks the Universitäts-Sternwarte Göttingen for support.

\section{References}

Altrock, R. C., November, L. J., Simon, G. W., Milkey, R. W., \& Worden, S. P. 1975, Sol. Phys., 43, 33

Bendlin, C., Volkmer, R., \& Kneer, F. 1992, A\&A, 257, 817

Bonet, J. A., Sobotka, M., \& Vázquez, M. 1995, A\&A, 296, 241

Brants, J. J. 1985, Sol. Phys., 98, 197

Brants, J. J., \& Zwaan, C. 1982, Sol. Phys., 80, 251

Brants, J. J., \& Steenbeek, C. M. 1985, Sol. Phys., 96, 229

Bray, R. J., \& Loughhead, R. E. 1964, Sunspots (London: Chapman and Hall)

Choudhuri, A. R. 1986, ApJ, 302, 809

de Boer, C. R. 1993, Ph.D. Thesis, Universität Göttingen

Degenhardt, D., \& Lites, B. W. 1993, ApJ, 404, 383
Deinzer, W., Hensler, G., Schüssler, M., \& Weisshaar, E. 1984, A\&A, 139,435

Hirzberger, J., \& Kneer, F. 2001, A\&A, 378, 1078

Hirzberger, J., Koschinsky, M., Kneer, F., \& Ritter, C. 2001, A\&A, 367,1011

Hirzberger, J., Bonet, J. A., Sobotka, M., Vázquez, M., \& Hanslmeier, A. 2002, A\&A, 383, 275

Hurlburt, N. E., \& Rucklidge, A. M. 2000, MNRAS, 314, 793

Keil, S. L., Balasubramaniam, K. S., Smaldone, L. A., \& Reger, B. 1999, ApJ, 510, 422

Keller, C. U. 1992, Nature, 359, 307

Keppens, R., \& Martínez Pillet, V. 1996, A\&A, 316, 229

Kneer, F. 1973, Sol. Phys., 28, 361

Knölker, M., \& Schüssler, M. 1988, A\&A, 202, 275

Krieg, J., Wunnenberg, M., Kneer, F., Koschinsky, M., \& Ritter, C. 1999, A\&A, 343, 983

Leka, K. D., \& Skumanich, A. 1998, ApJ, 507, 454

Lites, B. W., Bida, T. A., Johannesson, A., \& Scharmer, G. B. 1991, ApJ, 373, 683

Montesinos, B., \& Thomas, J. H. 1997, Nature, 390, 485

Muglach, K., Solanki, S. K., \& Livingston, W. C. 1994, in Solar Surface Magnetism, ed. R. J. Rutten, \& C. J. Schrijver (Dordrecht: Kluwer), 127

Muller, R., \& Roudier, Th. 1992, Sol. Phys., 141, 27

Parker, E. N. 1979, ApJ, 230, 905

Parker, E. N. 1992, ApJ, 390, 290

Petrovay, K., \& Moreno Insertis, F. 1997, ApJ, 485, 398

Rimmele, T. R. 1997, ApJ, 490, 458

Roudier, Th., Bonet, J. A., \& Sobotka, M. 2002, A\&A, 395, 249

Rucklidge, A. M., Schmidt, H. U., \& Weiss, N. O. 1995, MNRAS, 273, 491

Schmidt, W., \& Balthasar, H. 1994, A\&A, 283, 241

Sobotka, M. 1997, in Advances in Physics of Sunspots, ed. B. Schmieder, J. C. del Toro Iniesta, \& M. Vázquez, ASP Conf. Ser., 118, 155

Sobotka, M., Bonet, J. A., \& Vázquez, M. 1994, ApJ, 426, 404

Sobotka, M., Vázquez, M., Bonet, J. A., Hanslmeier, A., \& Hirzberger, J. 1999, ApJ, 511, 436

Spruit, H. C. 1979, Sol. Phys., 61, 363

Steiner, O., Grossmann-Doerth, U., Knölker, M., \& Schüssler, M. 1998, ApJ, 495, 468

Sütterlin, P. 1998, A\&A, 333, 305

Sütterlin, P., Thim, F., \& Schröter, E. H. 1994, in Solar Magnetic Fields, ed. M. Schüssler, \& W. Schmidt (Cambridge Univ. Press), 213

Sütterlin, P., Schröter, E. H., \& Muglach, K. 1996, Sol. Phys., 164, 311

Title, A. M., Tarbell, T. D., \& Topka, K. P. 1987, ApJ, 317, 892

Vázquez, M. 1973, Sol. Phys., 31, 377

Wang, H., \& Zirin, H. 1992, Sol. Phys., 140, 41

Westendorp Plaza, C., del Toro Iniesta, J. C., Ruiz Cobo, B., \& Martínez Pillet, V. 2001, ApJ, 547, 1148

Yi, Z., \& Molowny Horas, R. L. 1992, in Proc. LEST Mini-Workshop, Software for Solar Image Processing, ed. Z. Yi, T. Darvann, \& R. Molowny Horas (Oslo: Inst. f. Theoretical Astrophys.), 69

Zwaan, C. 1985, Sol. Phys., 100, 397

Zwaan, C. 1992, in Sunspots: Theory and Observations, ed. J. H. Thomas, \& N. O. Weiss (Dordrecht: Kluwer), 75 